\title{
Prognostic Indicators for Dengue Infection Severity
}

\author{
Surangrat Pongpana, b, d, Apichart Wisitwong ${ }^{\mathrm{c}}$, Chamaiporn Tawichasri ${ }^{\mathrm{a}}$, \\ Jayanton Patumanond ${ }^{\mathrm{a}}$
}

\begin{abstract}
Background: Symptomatic dengue infection can be classified into 3 patterns based on their severity; dengue fever (DF), dengue hemorrhagic fever (DHF) and dengue shock syndrome (DSS). In clinical practice, the diagnosis and management are based on clinical findings and abnormal initial laboratory tests. We conducted the present study to explore a set of parameters, preferable routine, or at least easy to investigate, that could be used as indicators for dengue infection severity.
\end{abstract}

Methods: A retrospective cohort study was conducted in three university-affiliated hospitals, one general hospital and two referral hospitals, in the northern part of Thailand. Patients were grouped into the three severity categories (DF, DHF, and DSS), using modified WHO criteria. Pre-defined prognostic indicators were compared. The prognostic indicators for dengue severity were analyzed by a multivariable polytomous logistic regression and presented with odds ratios.

Results: From 777 patients overall, 391 were classified as DF, 296 with DHF, and 90 with DSS. The characteristics that increased the risk of DHF were; age $>6$ year $(\mathrm{OR}=1.85)$, hepatomegaly $(\mathrm{OR}=$ $3.49)$, any bleeding episodes $(\mathrm{OR}=1.43)$, white cell count $>5,000$ / $\mu \mathrm{L}(\mathrm{OR}=1.80)$, and platelet $\leq 100,000 / \mu \mathrm{L}(\mathrm{OR}=3.72)$. The characteristics that increase the risk of DSS were; hepatomegaly (OR $=43.44)$, any bleeding episodes $(\mathrm{OR}=5.58)$, pulse pressure $\leq 20$ $\mathrm{mmHg}(\mathrm{OR}=19.09), \mathrm{SBP}<90 \mathrm{mmHg}(\mathrm{OR}=2.45)$, hematocrit $>$ $40 \%(\mathrm{OR}=1.88)$, white cell count $>5,000 / \mu \mathrm{L}(\mathrm{OR}=2.36)$, and

Manuscript accepted for publication March 13, 2013

${ }^{\mathrm{a}}$ Clinical Epidemiology Program, Faculty of Medicine, Chiang Mai University, Chiang Mai 50200, Thailand

${ }^{\mathrm{b}}$ Department of Occupational Medicine, Phrae Hospital, Phrae, 54000, Thailand

${ }^{\mathrm{c}}$ Department of Social Medicine, Sawanpracharak Hospital, Nakorn Sawan, 60000, Thailand

${ }^{\mathrm{d}}$ Corresponding author: Surangrat Pongpan, Clinical Epidemiology Program, Faculty of Medicine, Chiang Mai University, Chiang Mai 50200, Thailand. Email: rang130214@gmail.com

doi: http://dx.doi.org/10.4021/ijcp73w platelet $\leq 100,000 / \mu \mathrm{L}(\mathrm{OR}=10.60)$.

Conclusions: The severity of dengue infection is significantly associated with some routine clinical parameters. These parameters may be used to develop a future scoring system to predict and manage dengue infection severity early in the course of the illness.

Keywords: Dengue infection; Dengue hemorrhagic fever; Dengue shock syndrome; Severity; Prognostic indicators; Clinical risk; Multivariable analysis

\section{Introduction}

Most of dengue infections are asymptomatic. Those with symptoms can be classified into 3 patterns, based on their severity; undifferentiated fever, dengue fever (DF) and dengue hemorrhagic fever (DHF) which if accompanied by shock, is called dengue shock syndrome (DSS) [1].

In clinical practice, the diagnosis and management are based on clinical findings and abnormal initial laboratory tests [2]. Other laboratory tests may be requested to confirm case, as being used in research. These tests take several days to weeks for the results and are not used in routine practice. They are therefore used mainly for epidemiological purposes $[3,4]$.

The causes of mortality in dengue infection are from prolonged shock, massive bleeding and fluid overload. The main problem leading to poor prognosis or death is not being diagnosed when presenting in critical conditions at the hospitals $[5,6]$.

Clinical risks and laboratory tests have been studied to forecast the severity of infection. These included gender [7], younger age [7], presence of hepatomegaly [8], abdominal pain [9], lethargy, cold hands and feet [10], abnormal bleeding episodes [11, 12], obesity or over-weight (in children) $[9,13]$, malnourishment [14], type 2 dengue infection [13], secondary infection [11], presence of ascites [8], plural effusion [8], leucopenia $(<4,000 / \mu \mathrm{L})[8]$, thrombocytopenia [10], hemo-concentration [11], rising SGOT and/or SGPT $[12,15]$, prolonged PTT [16], prolonged PT [17], positive 
Table 1. Grading Dengue Infection Severity (Modified WHO 1997)

\begin{tabular}{llll}
\hline DF/DHF & Grade & Symptoms & Laboratory \\
\hline DF* & & $\begin{array}{l}\text { Fever with two or more of the following signs: } \\
\text { headache, retro-orbital pain, myalgia, arthralgia } \\
\text { plus positive tourniquet test }\end{array}$ & $\begin{array}{l}\text { Leucopenia occasionally } \\
\text { Thrombocytopenia, may be } \\
\text { present, no evidence of plasma } \\
\text { loss }\end{array}$ \\
DHF & I & Above signs plus positive tourniquet test & Thrombocytopenia $\leq 100,000 /$ \\
DHF & II & Above signs plus spontaneous bleeding & $\mu \mathrm{L}$, hematocrit rise $\geq 20 \%$
\end{tabular}

DF: dengue fever; DHF: dengue hemorrhagic fever; *Modified WHO criteria; ${ }^{*}$ DHF Grade III and IV are also called as dengue shock syndrome (DSS).

of the D-dimer test [18], and gallbladder wall thickening (measured by ultrasound) [19]. It is obvious that many parameters above are not used as routine practice in general hospitals. Many parameters require days to weeks for final reports. Some reports have very few sample sizes and some are descriptive or case series.

We conducted the present study to explore a set of parameters, preferable routine, or at least easy to investigate, that could be used as indicators for dengue infection severity. These indicators may be used to forecast dengue infection severity at the time the patients arrive at out-patient or emergency departments of the hospitals.

\section{Materials and Methods}

\section{Patients}

Three university-affiliated hospitals were selected to represent the study domain, one general hospital (in Kamphaeng Phet) and two referral hospitals (in Nakorn Sawan and in Uttaradit). Medical files of patients admitted between 2007 and 2010 were searched under the following ICD-10s; A90Dengue fever, A91-Dengue hemorrhagic fever, and A910Dengue hemorrhagic fever with shock.

\section{Criteria for dengue severity}

The severity of dengue infection is classified into different grades using modified WHO criteria [1] (Table 1).

\section{Prognostic indicators}

Patient characteristics of interest included: 1). Demographic: gender and age; 2). Mode of Presentation: presence of hepatomegaly, headache, myalgia, vomiting, cough, abdominal pain, rash, pleural effusion, petechiae, and any bleeding episodes; 3). Hemodynamic: pulse pressure, SBP (systolic blood pressure), and DBP (diastolic blood pressure); 4). Hematological: hematocrit, hemoglobin, white cell count, lymphocytes, neutrophils, and platelet; 5). Biochemical: SGOT (serum glutamic oxaloacetic transaminase), SGPT (serum glutamic pyruvic transaminase), PT (prothrombin time), and PTT (partial thromboplastin time).

\section{Data analysis}

Different distribution of patient characteristics among the three severity categories (DF, DHF, and DSS) was analyzed by exact probability test, analysis of variance (ANOVA), or ANOVA by rank based on types of variables. Complete data were used to screen for selected important parameters. Significant parameters were analyzed by the multivariable polytomous logistic regression to identify a set of significant characteristics associated with severity. Multiple imputation techniques were applied to clinical variables with missing values in the last step of analysis.

\section{Ethical approval}

The study protocol was approved by The Research Ethics 
Table 2. Demographic and Clinical Profiles by Types of Dengue Infection

\begin{tabular}{|c|c|c|c|c|}
\hline \multirow{2}{*}{ Patient characteristics } & DF $(n=391)$ & DHF $(n=296)$ & $\operatorname{DSS}(\mathrm{n}=90)$ & \multirow{2}{*}{ P-value* } \\
\hline & Mean \pm SD & Mean \pm SD & Mean \pm SD & \\
\hline \multicolumn{5}{|l|}{ Demographic } \\
\hline Male (n, \%) & $185(47.3)$ & $153(51.7)$ & $38(42.2)$ & 0.247 \\
\hline Age (year) & $9.5 \pm 3.4$ & $10.1 \pm 3.1$ & $9.0 \pm 3.5$ & 0.009 \\
\hline \multicolumn{5}{|l|}{ Mode of presentation } \\
\hline Hepatomegaly (n, \%) & $8(2.1)$ & $26(8.8)$ & $55(61.1)$ & $<0.001$ \\
\hline Headache (n, \%) & $228(58.3)$ & $145(49.0)$ & $35(38.9)$ & $<0.001$ \\
\hline Myalgia (n, \%) & $84(21.5)$ & $30(10.1)$ & $12(13.3)$ & 0.001 \\
\hline Vomiting $(\mathrm{n}, \%)$ & $244(62.4)$ & $216(73.0)$ & $56(62.2)$ & 0.009 \\
\hline Cough (n, \%) & $120(30.7)$ & $94(31.8)$ & $32(35.6)$ & 0.751 \\
\hline Abdominal pain (n, \%) & $160(40.9)$ & $181(61.2)$ & $60(66.7)$ & $<0.001$ \\
\hline Rash (n, \%) & $166(42.5)$ & $132(44.6)$ & $26(28.9)$ & 0.026 \\
\hline Pleural effusion & $0(0)$ & $20(6.8)$ & $34(37.8)$ & $<0.001$ \\
\hline Petechiae (n, \%) & $27(6.9)$ & $26(8.8)$ & $14(15.6)$ & 0.039 \\
\hline Any bleeding episodes (n, \%) & $73(18.7)$ & $80(27.0)$ & $51(56.7)$ & $<0.001$ \\
\hline \multicolumn{5}{|l|}{ Hemodynamic } \\
\hline $\mathrm{SBP}(\mathrm{mmHg})$ & $97.0 \pm 9.2$ & $96.9 \pm 10.1$ & $91.3 \pm 10.3$ & $<0.001$ \\
\hline $\mathrm{DBP}(\mathrm{mmHg})$ & $58.4 \pm 7.7$ & $58.2 \pm 8.6$ & $58.6 \pm 8.4$ & 0.356 \\
\hline Pulse pressure (mmHg) & $33.1 \pm 6.7$ & $32.1 \pm 7.6$ & $24.0 \pm 7.7$ & $<0.001$ \\
\hline \multicolumn{5}{|l|}{ Hematological } \\
\hline Hematocrit (\%) & $38.8 \pm 4.5$ & $40.5 \pm 4.8$ & $42.4 \pm 5.4$ & $<0.001$ \\
\hline Hemoglobin $(\mathrm{g} / \mathrm{dl})$ & $12.7 \pm 1.5$ & $13.4 \pm 1.5$ & $14.1 \pm 1.8$ & $<0.001$ \\
\hline White cell count $(/ \mu \mathrm{L})^{* *}$ & $3,348.0 \pm 1.6$ & $3,737.5 \pm 1.7$ & $4,773.4 \pm 1.7$ & $<0.001$ \\
\hline Lymphocytes (\%) & $44.9 \pm 17.3$ & $40.8 \pm 15.7$ & $40.0 \pm 16.3$ & $<0.001$ \\
\hline Neutrophils (\%) & $44.7 \pm 18.8$ & $49.5 \pm 18.1$ & $48.9 \pm 17.6$ & $<0.001$ \\
\hline Platelet $(/ \mu \mathrm{L})^{* *}$ & $106,084.3 \pm 1.8$ & $62,533.8 \pm 2.2$ & $36,074 \pm 2.3$ & $<0.001$ \\
\hline \multicolumn{5}{|l|}{ Biochemical } \\
\hline SGOT $(\mathrm{U} / \mathrm{L})^{* *}$ & $84.2 \pm 2.4$ & $158.1 \pm 2.4$ & $219.4 \pm 3.7$ & $<0.001$ \\
\hline $\mathrm{SGPT}(\mathrm{U} / \mathrm{L})^{* *}$ & $42.1 \pm 2.3$ & $60.6 \pm 2.4$ & $78.7 \pm 3.7$ & 0.005 \\
\hline PT $(\mathrm{sec})$ & $12.0 \pm 1.5$ & $12.1 \pm 1.8$ & $15.5 \pm 9.5$ & 0.159 \\
\hline PTT (sec) & $30.4 \pm 5.4$ & $42.0 \pm 10.0$ & $44.7 \pm 12.3$ & 0.011 \\
\hline \multicolumn{5}{|l|}{ Case management } \\
\hline Inbound referral (n, \%) & $43(11.0)$ & $78(26.4)$ & $48(53.3)$ & $<0.001$ \\
\hline \multicolumn{5}{|l|}{ Discharged } \\
\hline Alive (n, \%) & $391(100.0)$ & $296(100.0)$ & $88(97.8)$ & 0.013 \\
\hline Outbound referral (n, \%) & $0(0)$ & $0(0)$ & $2(2.2)$ & 0.013 \\
\hline In hospital dead (n, \%) & $0(0)$ & $0(0)$ & $0(0)$ & 0.013 \\
\hline
\end{tabular}

Dengue fever (DF); dengue hemorrhagic fever (DHF); dengue shock syndrome (DSS); *P-value from exact probability test or analysis of variance (ANOVA) or ANOVA by rank; ** geometric mean and standard deviation (SD); SGOT: serum glutamic oxaloacetic transaminase; SGPT: serum glutamic pyruvic transaminase; PT: prothrombin time; PTT: partial thromboplastin time. 
Committee, Faculty of Medicine, Chiang Mai University, and the research ethics committee of the three hospitals.

\section{Results}

There were 777 patients overall, 391 with DF, 296 with DHF, and 90 with DSS. By univariable analysis, the three severity groups were similar in gender, age, presence of vomiting, cough, rash and SBP. The characteristics that were different by severity categories were; hepatomegaly, headache, myalgia, abdominal pain, pleural effusion, petechiae, any bleeding episodes, SBP, pulse pressure, hematocrit, hemoglobin, white cell count, lymphocytes, neutrophils, platelet, SGOT, SGPT, PT, and PTT (Table 2).

Under multivariable polytomous logistic regression, characteristics that increased the risk of DHF were; age $>6$ year $(\mathrm{OR}=1.85)$, hepatomegaly $(\mathrm{OR}=3.49)$, any bleeding episodes $(\mathrm{OR}=1.43)$, white cell count $>5,000 / \mu \mathrm{L}(\mathrm{OR}=$ $1.80)$, and platelet $\leq 100,000 / \mu \mathrm{L}(\mathrm{OR}=3.72)$. The characteristics that increase the risk of DSS were; hepatomegaly (OR $=43.44)$, any bleeding episodes $(\mathrm{OR}=5.58)$, pulse pressure $\leq 20 \mathrm{mmHg}(\mathrm{OR}=19.09), \mathrm{SBP}<90 \mathrm{mmHg}(\mathrm{OR}=2.45)$, hematocrit $>40 \%(\mathrm{OR}=1.88)$, white cell count $>5,000 / \mu \mathrm{L}$ $(\mathrm{OR}=2.36)$, and platelet $\leq 100,000 / \mu \mathrm{L}(\mathrm{OR}=10.60)$ (Table 3).

\section{Discussion}

\section{Demographic}

Some previous studies reported more risk of shock and death in females [7, 20], due to more health seeking behavior to traditional practitioners [21]. Immune responses in females may be more sensitive to cytokines secretion, affecting higher degree of plasma leakage [21, 22]. However, our study and one other study [23] did not find gender as a risk of dengue infection severity.

Patients $>6$ years were at risk for DHF and DSS in some studies [8,9] from many factors such as serological coverage and mutation [24]. It was observed that children $<10$ years are more prone to increased vascular permeability, leading to shock $[9,22]$.

\section{Mode of presentation}

Hepatomegaly was a strong risk factors in our study, similar to many studies in the past $[10,25]$. Although moderate liver enlargement is a normal response to dengue infection [26], it is more associated with DHF and DSS compared to DF $[27,28]$.

Abdominal pain was reported as another prognostic factor $[9,10,25]$. It could be caused by gastrointestinal bleeding 
and/or hepatomegaly. Other studies proposed that during the shock or pre-shock state, blood supply to visceral organs was reduced causing tissue hypoxia followed by abdominal pain [9]. This was not found in our study and one other study [29].

Pleural effusion was reported in some studies [8, 12, 30, 31 ], as it was found most commonly in dengue infection, associated with plasma leakage, leading to hypovolemic shock [1]. This was not found in our study.

In our and many studies, any bleeding episodes were also a prognostic factor $[8,11,12]$. This is associated with thrombocytopenia found in severe dengue infection. Platelet $<50,000 / \mu \mathrm{L}$ is associated with severe bleeding [32]. Bleeding may also caused by platelet dysfunction, vasculopathy and/or coagulopathy related to severe bleeding which may lead to death [33]. However some study did not find such relationship [9].

\section{Hemodynamic}

As expected, SBP $<90 \mathrm{mmHg}$ and pulse pressure $\leq 20$ $\mathrm{mmHg}$ are prognostic factors for DSS [34, 35]. WHO has used lowered SBP or narrow pulse pressure as criteria to classify dengue infection into different severity grades [1].

\section{Hematological}

Hematocrit $>40 \%$ is a prognostic factor for dengue infection severity particularly DSS, confirming the findings from many studies $[10,12,28]$. Vasculopathy in dengue infection causes increased vascular permeability, leading to hemoconcentration and shock $[33,36]$. Hemo-concentration (hematocrit $\geq 20 \%$ of initial value) is one of the WHO criteria to diagnose DHF. Only few study reported no such association [8].

Similar to many studies, platelet $\leq 100,000 / \mu \mathrm{L}$ increased the risk of severity $[10,17,28]$. Platelet decreased rapidly before patients enter the state of shock. WHO also used platelets to categorize dengue infection into DHF grade I-IV [1]. Low platelet was explained by bone marrow suppression and immune-response induced platelet destruction by liver and spleen $[5,33,36]$.

Some study concluded that white cell count $>5,000 / \mu \mathrm{L}$ is a prognostic factor for dengue severity $[6,15]$ while others found leucopenia $[8,12]$. Normoleukocytosis or mild leukocytosis may be found in early dengue infection. When body temperature declines, most patients experienced leucopenia from bone marrow suppression [33]. Stress in accompanied with shock may somehow cause leukocytosis [6].

\section{Biochemical}

Elevated liver enzymes (SGOT and SGPT) are known as prognostic factors for DSS [15, 37]. When liver cells are infected, SGOT level is higher than SGPT particularly in se- vere cases [36]. The averaged SGOT and SGPT are higher in DHF than in DF [2]. Prolonged PTT and prolonged PT are prognostic factors for DHF and DSS [16, 17]. However, the present study and one other study [11] did not find such association. Elevated liver enzymes, prolonged PT and prolonged PTT normally occur after shock [11], which is too late to be used as prognostic indicators. Furthermore, SGOT, SGPT, PT, PTT are not routinely investigated in many hospitals, limiting their use in prognostic indicators.

Significant clinical parameters associated with severity of dengue infection may be used further in the development of a prognostic score, to forecast disease severity in patients suspected of dengue infection. If feasible, this forecasting may help early case management and reduce the overall morbidity and mortality.

Retrospective nature of the data limited its completeness of all clinical parameters of interests. However, we believe that information not investigated nor recorded in routine practice, are of limited values in perspectives of forecasting or prediction. With our relatively large sample size and multiple imputation techniques used for missing parameters, we believe that our results are likely to be valid.

\section{Conclusions}

The severity of dengue infection is significantly associated with some routine clinical parameters. These parameters may be used to develop a future scoring system to predict and manage dengue infection severity early in the course of the illness.

\section{Acknowledgement}

The authors wish to thank the authorities and the research committee of the 3 hospitals for their collaboration in data collection.

\section{Grant Support}

This report was partially supported by the grant from The Faculty of Medicine, Chiang Mai University.

\section{Financial Disclosures and Conflict of Interests}

None declared.

\section{References}

1. World Health Organization. Dengue hemorrhagic fever: diagnosis, treatment, prevention and control. Geneva: 
WHO, 1997.

2. Kalayanarooj S, Vaughn DW, Nimmannitya S, Green S, Suntayakorn S, Kunentrasai N, Viramitrachai W, et al. Early clinical and laboratory indicators of acute dengue illness. J Infect Dis. 1997;176(2):313-321.

3. Guzman MG, Kouri G. Dengue diagnosis, advances and challenges. Int J Infect Dis. 2004;8(2):69-80.

4. Ahmed A. Diagnosing dengue fever. Infectious Diseases Journal of Pakistan. 2005:129-132.

5. Chuansumrit A, Tangnararatchakit K. Pathophysiology and management of dengue hemorrhagic fever. Transfus Altern Transfus Med. 2006;8(Suppl 1):S3-11.

6. Buranakitjaroen P. Hypertension audit in clinical practice based in Thailand (HABIT). J Med Assoc Thai. 2011;94(Suppl 1):S57-65.

7. Anders KL, Nguyet NM, Chau NV, Hung NT, Thuy TT, Lien le B, Farrar J, et al. Epidemiological factors associated with dengue shock syndrome and mortality in hospitalized dengue patients in Ho Chi Minh City, Vietnam. Am J Trop Med Hyg. 2011;84(1):127-134.

8. Gupta V, Yadav TP, Pandey RM, Singh A, Gupta M, Kanaujiya P, Sharma A, et al. Risk factors of dengue shock syndrome in children. J Trop Pediatr. 2011;57(6):451-456.

9. Junia J, Garna H, Setiabudi D. Clinical risk factors for dengue shock syndrome in children. Paediatr Indones. 2007;47(1):7-11.

10. Pham TB, Nguyen TH, Vu TQ, Nguyen TL, Malvy D. [Predictive factors of dengue shock syndrome at the children Hospital No. 1, Ho-chi-Minh City, Vietnam]. Bull Soc Pathol Exot. 2007;100(1):43-47.

11. Tantracheewathorn T, Tantracheewathorn S. Risk factors of dengue shock syndrome in children. J Med Assoc Thai. 2007;90(2):272-277.

12. Chacko B, Subramanian G. Clinical, laboratory and radiological parameters in children with dengue fever and predictive factors for dengue shock syndrome. J Trop Pediatr. 2008;54(2):137-140.

13. Pichainarong N, Mongkalangoon N, Kalayanarooj S, Chaveepojnkamjorn W. Relationship between body size and severity of dengue hemorrhagic fever among children aged 0-14 years. Southeast Asian J Trop Med Public Health. 2006;37(2):283-288.

14. Kalayanarooj S, Nimmannitya S. Is dengue severity related to nutritional status? Southeast Asian J Trop Med Public Health. 2005;36(2):378-384.

15. Azin FR, Goncalves RP, Pitombeira MH, Lima DM, Branco IC. Dengue: profile of hematological and biochemical dynamics. Rev Bras Hematol Hemoter. 2012;34(1):36-41.

16. Shah I, Deshpande GC, Tardeja PN. Outbreak of dengue in Mumbai and predictive markers for dengue shock syndrome. J Trop Pediatr. 2004;50(5):301-305.

17. Chuansumrit A, Puripokai C, Butthep P, Wongtiraporn
W, Sasanakul W, Tangnararatchakit K, Chunhakan S, et al. Laboratory predictors of dengue shock syndrome during the febrile stage. Southeast Asian J Trop Med Public Health. 2010;41(2):326-332.

18. Bongsebandhu-phubhakdi C, Hemungkorn M, Thisyakorn U, Thisyakorn C. Risk factors influencing severity in pediatric dengue infection. Asian Biomed. 2008;2(5):409-413.

19. Colbert JA, Gordon A, Roxelin R, Silva S, Silva J, Rocha C, Harris E. Ultrasound measurement of gallbladder wall thickening as a diagnostic test and prognostic indicator for severe dengue in pediatric patients. Pediatr Infect Dis J. 2007;26(9):850-852.

20. Phuong CX, Nhan NT, Kneen R, Thuy PT, van Thien C, Nga NT, Thuy TT, et al. Clinical diagnosis and assessment of severity of confirmed dengue infections in Vietnamese children: is the world health organization classification system helpful? Am J Trop Med Hyg. 2004;70(2):172-179.

21. Guha-Sapir D, Schimmer B. Dengue fever: new paradigms for a changing epidemiology. Emerg Themes Epidemiol. 2005;2(1):1.

22. Halstead SB. Epidemiology of dengue and dengue hemorrhagic fever. In: Gubler DJ, Kuno G, eds. Dengue and dengue hemorrhagic fever. London: Cambridge University, 1997:23-44.

23. Ahmed FU, Mahmood CB, Jhulan Das Sharma, Hoque SM, Zaman R, Hasan MS. Dengue and dengue haemorrhagic fever in children during the 2000 outbreak in Chittagong, Bangladesh. Dengue Bull. 2001;25:33-39.

24. Wichmann O, Hongsiriwon S, Bowonwatanuwong C, Chotivanich K, Sukthana Y, Pukrittayakamee S. Risk factors and clinical features associated with severe dengue infection in adults and children during the 2001 epidemic in Chonburi, Thailand. Trop Med Int Health. 2004;9(9):1022-1029.

25. Falconar AK, Romero-Vivas CM. Simple Prognostic Criteria can Definitively Identify Patients who Develop Severe Versus Non-Severe Dengue Disease, or Have Other Febrile Illnesses. J Clin Med Res. 2012;4(1):33-44.

26. Seneviratne SL, Malavige GN, de Silva HJ. Pathogenesis of liver involvement during dengue viral infections. Trans R Soc Trop Med Hyg. 2006;100(7):608-614.

27. Kittigul L, Pitakarnjanakul P, Sujirarat D, Siripanichgon $\mathrm{K}$. The differences of clinical manifestations and laboratory findings in children and adults with dengue virus infection. J Clin Virol. 2007;39(2):76-81.

28. Shah GS, Islam S, Das BK. Clinical and laboratory profile of dengue infection in children. Kathmandu Univ Med J (KUMJ). 2006;4(1):40-43.

29. Al-Araimi H, Al-Jabri A, Mehmoud A, Al-Abri S. Dengue Haemorrhagic Fever presenting as Acute Abdomen. Sultan Qaboos Univ Med J. 2011;11(2):265-268.

30. Alam K, Sulaiman SAS, Shafie AA, Yusuf E. Clinical 
manifestations and laboratory profile of dengue fever among the patient's general Hospital, Penang. Arch Pharma Pract. 2010;1(1):25-29.

31. Karyanti MR. Clinical manifestations and hematological and serological findings in children with dengue infection. Paediatr Indones. 2011;51(3):157-162.

32. Jr GDR, Torno LL. Association of leukocyte and thrombocyte counts as a predictor of bleeding outcomes among dengue patients. Philippine Journal of Microbiology and Infectious Diseases. 2007;36(1-2):33-38.

33. Srichaikul T, Nimmannitya S. Haematology in dengue and dengue haemorrhagic fever. Baillieres Best Pract
Res Clin Haematol. 2000;13(2):261-276.

34. Morens DM, Fauci AS. Dengue and hemorrhagic fever: a potential threat to public health in the United States. JAMA. 2008;299(2):214-216.

35. Simmons CP, Farrar JJ, Nguyen v V, Wills B. Dengue. N Engl J Med. 2012;366(15):1423-1432.

36. Lei HY, Yeh TM, Liu HS, Lin YS, Chen SH, Liu CC. Immunopathogenesis of dengue virus infection. J Biomed Sci. 2001;8(5):377-388.

37. Mohan B, Patwari AK, Anand VK. Hepatic dysfunction in childhood dengue infection. J Trop Pediatr. 2000;46(1):40-43. 